\title{
Dynamics of Symmetry-Breaking Stacking Boundaries in Bilayer $\mathrm{MoS}_{2}$
}

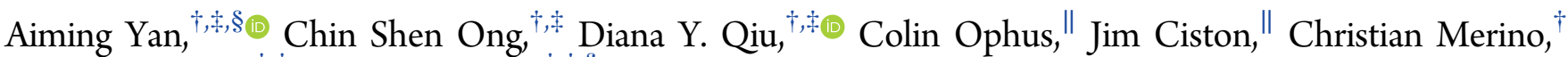 \\ Steven G. Louie, ${ }^{\dagger, \ddagger}$ and Alex Zettl ${ }^{*}, \dagger, \S$ \\ ${ }^{\dagger}$ Department of Physics, University of California, Berkeley, California 94720, United States \\ ${ }^{\ddagger}$ Materials Sciences Division, Lawrence Berkeley National Laboratory, Berkeley, California 94720, United States \\ ${ }^{\S}$ Kavli Energy NanoSciences Institute at the University of California, Berkeley and the Lawrence Berkeley National Laboratory, \\ Berkeley, California 94720, United States \\ "National Center for Electron Microscopy, Molecular Foundry, Lawrence Berkeley National Laboratory, Berkeley, California 94720, \\ United States
}

\section{Supporting Information}

ABSTRACT: Crystal symmetry of two-dimensional (2D) materials plays an important role in their electronic and optical properties. Engineering symmetry in $2 \mathrm{D}$ materials has recently emerged as a promising way to achieve novel properties and functions. The noncentrosymmetric structure of monolayer transition metal dichalcogenides (TMDCs), such as molybdenum disulfide $\left(\mathrm{MoS}_{2}\right)$, has allowed for valley control via circularly polarized optical excitation. In bilayer TMDCs, inversion symmetry can be controlled by varying the stacking sequence, thus providing a pathway to engineer valley selectivity. Here, we report the in situ integration of $\mathrm{AA}^{\prime}$ and $\mathrm{AB}$ stacked bilayer $\mathrm{MoS}_{2}$ with different inversion symmetries by creating atomically sharp stacking boundaries between the differently stacked domains, via thermal stimulation and electron irradiation, inside an atomic-resolution scanning transmission electron microscopy. The setup enables us to track the formation and atomic motion of the stacking boundaries in real time and with ultrahigh resolution which enables in-depth analysis on the atomic structure at the boundaries. In conjunction with density functional theory calculations, we establish the dynamics of the boundary nucleation and expansion and further identify metallic boundary states. Our approach provides a means to synthesize domain boundaries with intriguing transport properties and opens up a new avenue for controlling valleytronics in nanoscale domains via real-time patterning of domains with different symmetry properties.

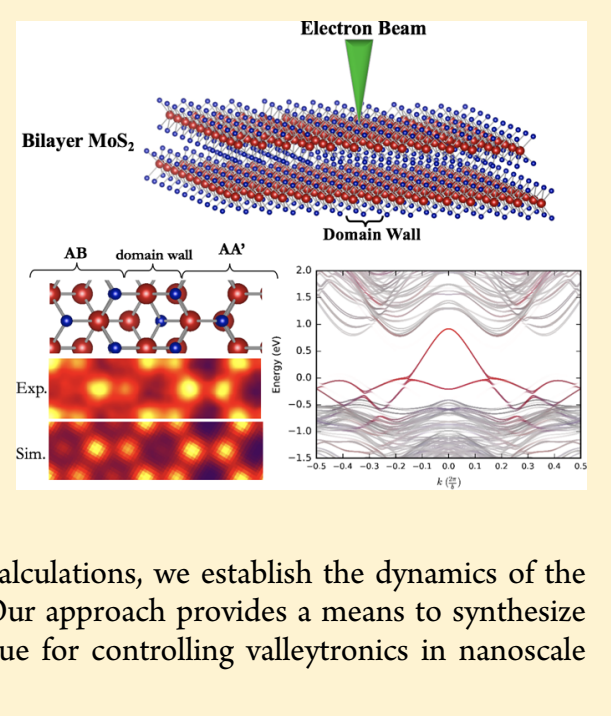

C rystal symmetry in a material dictates its physical properties. Manipulating its symmetry provides a pathway to achieve novel and unusual functionalities. This is particularly relevant for two-dimensional (2D) materials, promising candidates for numerous applications, including next-generation flexible electronics. In bilayer graphene, for example, symmetry-inversion at a "soliton" stacking boundary between $\mathrm{AB}$ and $\mathrm{BA}$ stacked regions ${ }^{1,2}$ leads to robust, topologically protected $1 \mathrm{D}$ conducting channels at the domain walls. ${ }^{3-5}$ In other $2 \mathrm{D}$ materials such as transition metal dichalcogenides (TMDCs), the noncentrosymmetric crystal structure of the monolayer form leads to valley selectivity and holds promise for applications in spintronics and valleytronics. ${ }^{6-9}$

One prototypical example of $2 \mathrm{D}$ TMDCs is monolayer $\mathrm{H}$ phase $\mathrm{MoS}_{2}$. Monolayer $\mathrm{H}$-phase $\mathrm{MoS}_{2}$ is noncentrosymmetric and has a lattice constant of $\boldsymbol{a}=3.16 \AA$ (Figure 1). The broken inversion symmetry, in addition to strong spin-orbit interactions, leads to the splitting of valence bands and spinvalley coupling, ${ }^{6}$ enabling valley selectivity when excited by circularly polarized light. ${ }^{7-9}$ However, inversion symmetry is restored in bilayer $2 \mathrm{H}$-phase $\mathrm{MoS}_{2}$ - a phase that is naturally found and most commonly observed (also known to adopt $\mathrm{AA}^{\prime}$ stacking sequence in Figure 1). To break the inversion symmetry in bilayer $\mathrm{MoS}_{2}$, a vertical electrical field can be applied. ${ }^{10}$ Another way to introduce asymmetry in bilayer and multilayer $\mathrm{MoS}_{2}$ is to engineer the stacking sequence by folding $^{11}$ or transferring ${ }^{12,13}$ exfoliated single-layer $\mathrm{MoS}_{2}$, or by using chemical synthesis methods such as chemical vapor transport $(\mathrm{CVT})^{14}$ and chemical vapor deposition (CVD). ${ }^{15-18}$ Two commonly observed stacking sequences in CVD/CVT grown $\mathrm{MoS}_{2}$ are $\mathrm{AA}^{\prime}$ stacking and $\mathrm{AB}$ stacking (a representative schematic of $A B$ stacking is shown in Figure 1), which are nearly energetically degenerate. ${ }^{15,19}$ Repeated $\mathrm{AB}$ stacking forms the $3 R$ phase of $\mathrm{MoS}_{2}$, in which centrosymmetry is broken throughout the bulk. A recent study shows that valleydependent spin polarization is realized in CVT synthesized 3Rphase bulk $\mathrm{MoS}_{2}$.

Received: August 22, 2017

Published: September 15, 2017 


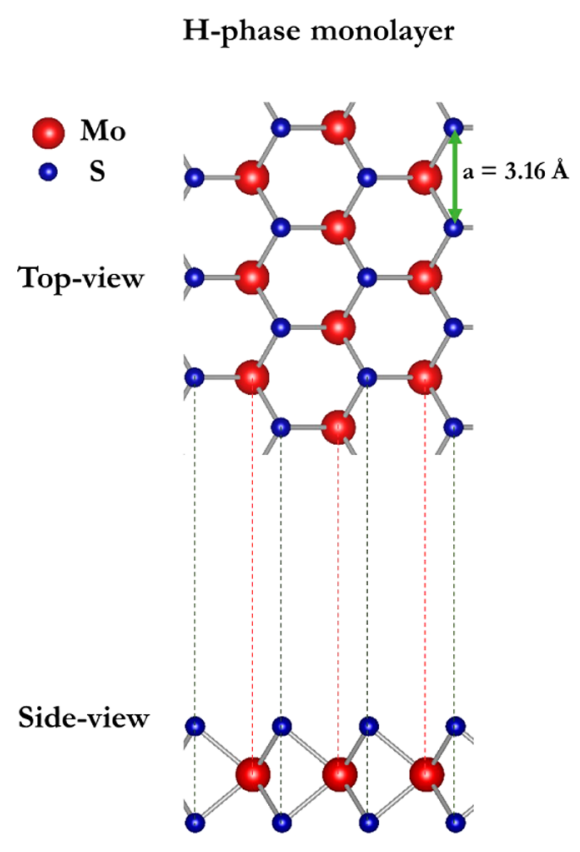

$$
\begin{gathered}
\text { AB stacking } \\
\text { (R phase) }
\end{gathered}
$$

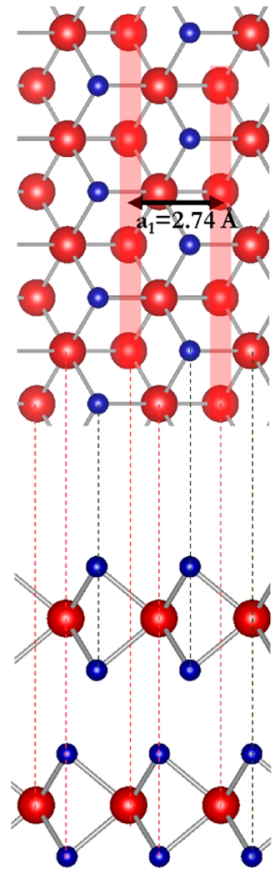

AA' stacking

(2H phase)

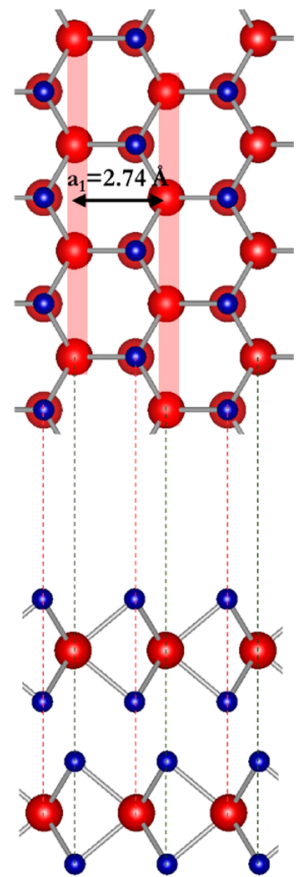

Figure 1. Ball-and-stick models for monolayer $H$-phase $\mathrm{MoS}_{2}$, and $\mathrm{AB}\left(R\right.$ phase) and $\mathrm{AA}^{\prime}\left(2 H\right.$ phase) stacked bilayer $\mathrm{MoS}_{2}$, viewed from the top and the side, with red balls representing Mo atoms and blue balls representing $S$ atoms. The lattice constant $(\boldsymbol{a})$ for monolayer $H$-phase $\mathrm{MoS}_{2}$ is $3.16 \AA$, and the Mo lattice distance in one layer $\operatorname{MoS}_{2}\left(a_{1}\right)$ is $2.74 \AA$, as indicated by red shadings in the schematics for both $\mathrm{AB}$ and $\mathrm{AA}$ ' bilayers.
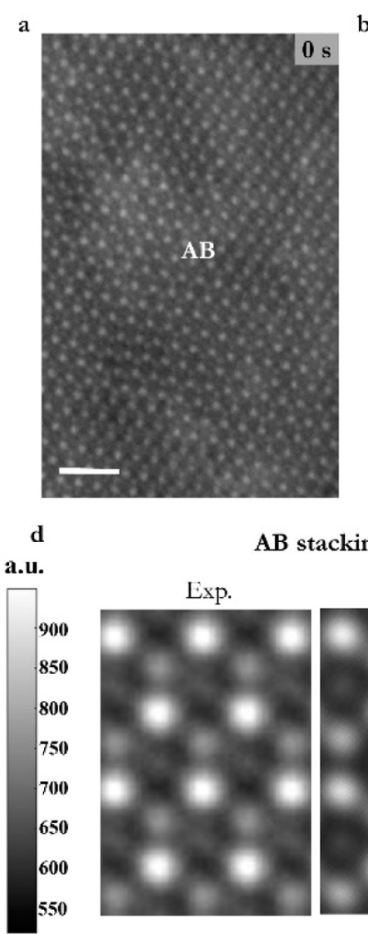
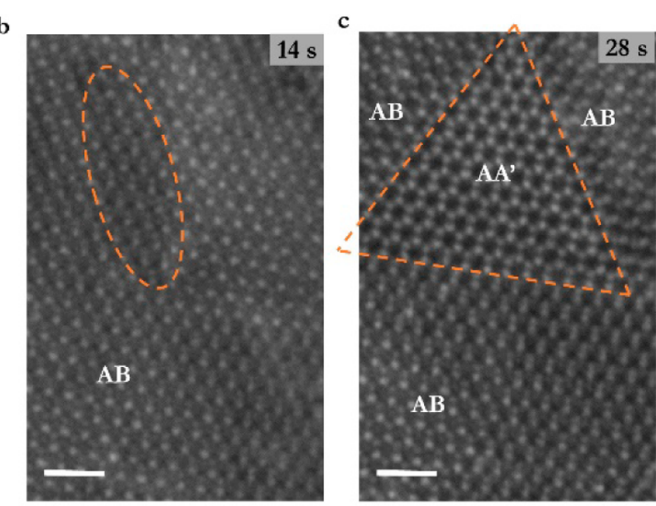

AA' stacking
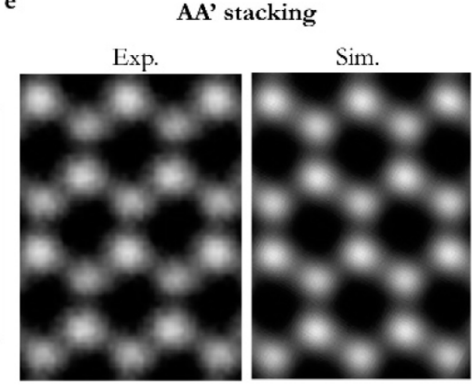

Figure 2. Stitching of $\mathrm{AB}$ and $\mathrm{AA}^{\prime}$ stacked regions with sharp stacking boundaries in bilayer $\mathrm{MoS}_{2}$. (a) High-resolution STEM ADF image taken at time $0 \mathrm{~s}$, showing $\mathrm{AB}$ stacked bilayer $\mathrm{MoS}_{2}$. (b) After $14 \mathrm{~s}$ of scanning in the same area, rearrangement of atoms occurred, as highlighted by the orange ellipse at the center of this $\mathrm{AB}$ stacked region. (c) High-resolution STEM ADF image showing a triangular AA' stacked region (highlighted by an orange triangle) surrounded by $A B$ stacked region. This image was taken $14 \mathrm{~s}$ after (b) and is from the same area as (b). (d, e) STEM ADF images from $\mathrm{AB}$ stacked region in (a) and $\mathrm{AA}^{\prime}$ stacked region in (c), respectively, with the intensity averaged over more than 10 unit cells of the same stacking sequence. The experimental results are compared to the multislice simulation ${ }^{26}$ of STEM ADF images of $\mathrm{AB}$ and $\mathrm{AA}^{\prime}$ stacked bilayer $\mathrm{MoS}_{2}$ under the same experimental imaging condition in (d) and (e), respectively. All images in (d) and (e) are plotted on the same absolute scale. Scale bars in (a)-(c) are $1 \mathrm{~nm}$. 


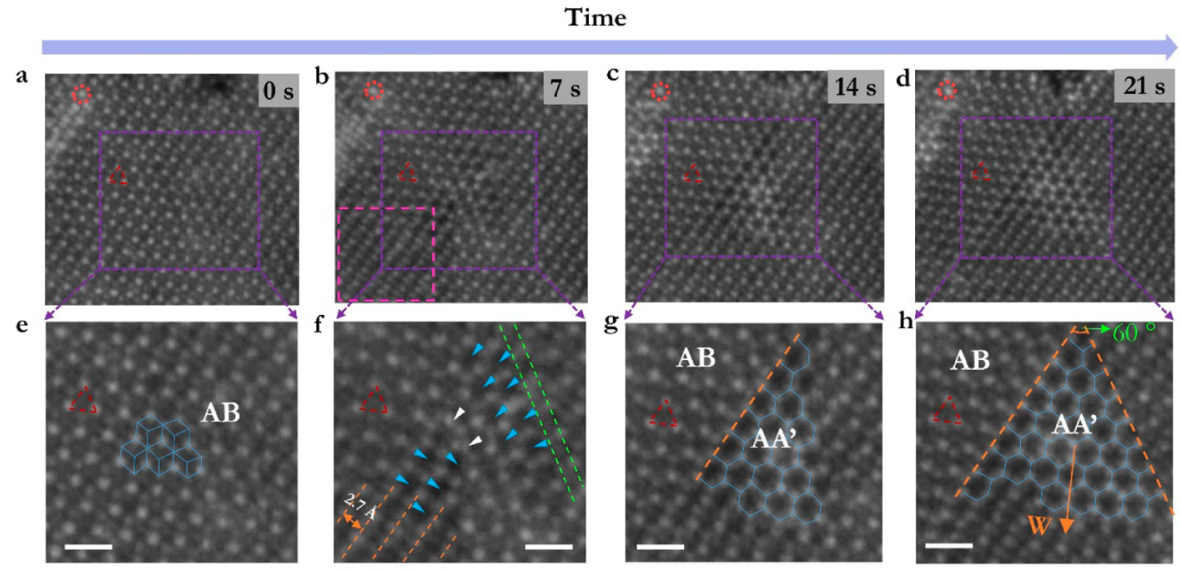

Figure 3. Nucleation and motion of the stacking boundaries in bilayer $\mathrm{MoS}_{2}$ at $400{ }^{\circ} \mathrm{C}$. (a) -(d) STEM ADF image series of structural change in bilayer $\mathrm{MoS}_{2}$ from the same area as time evolves from left to right. Red circles and triangles in these images highlight the same atoms and lattice points in this area. (e) $-(\mathrm{h})$ Enlarged images of the area highlighted with purple squares in (a) $-(\mathrm{d})$, respectively. (e) At $t=0 \mathrm{~s}, \mathrm{AB}$ stacked bilayer $\mathrm{MoS}_{2}$ with the lattice points connected by blue lines. The S-S columns from the top layer occupy the hollow centers of the hexagonal lattices in this image. (f) At $t=7 \mathrm{~s}$, local atomic rearrangement starts to occur, which triggers the nucleation of $\mathrm{AA}^{\prime}$ stacked domain. Large-area linear features with spacing $2.74 \AA$ (orange lines) are due to the interlayer lateral shift between the top and bottom $\mathrm{MoS}_{2}$ layers. Other structural changes are also observed, such as extra atoms (white arrowheads) and local shrinkage of projected Mo-Mo distance (green lines). ( $\mathrm{g}$ ) At $t=14 \mathrm{~s}, \mathrm{AA}^{\prime}$ stacked region grows to $\sim 2.88 \mathrm{~nm}^{2}$. (h) At $t=21 \mathrm{~s}, \mathrm{AA}^{\prime}$ stacked region grows to $4.75 \mathrm{~nm}^{2}$ as outlined by the blue hexagons. The angle between the two straight stacking boundaries is $60^{\circ}$. The growth direction for the $\mathrm{AA}^{\prime}$ stacked region is now along vector $\boldsymbol{W}$. Scale bars in (e)-(h) are $5 \AA$.

In this work, we demonstrate a novel way in which the symmetry of bilayer $\mathrm{MoS}_{2}$ can be engineered via the creation of nanoscale stacking boundaries that separate domains with different inversion symmetries. We show that, in bilayer $\mathrm{MoS}_{2}$, the transition from $\mathrm{AB}$ to $\mathrm{AA}^{\prime}$ stacking or from $\mathrm{AA}^{\prime}$ to $\mathrm{AB}$ stacking can be realized by heating and electron-irradiating the $\mathrm{AA}^{\prime}$ or $\mathrm{AB}$ stacked bilayer $\mathrm{MoS}_{2}$ inside an aberration-corrected scanning transmission electron microscope (STEM). By using in situ atomic-resolution STEM annular-dark field (ADF) imaging, we identify atomically sharp stacking boundaries between these two differently stacked domains. Combined with first-principles density functional theory (DFT) calculations, we discuss the atomic-scale dynamics of the domain nucleation and growth. DFT calculation also reveals the existence of highly localized metallic states at the domain boundaries.

Bilayer $\mathrm{MoS}_{2}$ studied here is grown on $\mathrm{SiO}_{2} / \mathrm{Si}$ substrates via a modified CVD method based on refs 20 and 21 (see the Supporting Information for details). The as-grown $\mathrm{MoS}_{2}$ flakes are then transferred to a Protochip heating TEM grid via the PMMA transfer method. ${ }^{22}$ The Protochip TEM grid holder has the ability to heat the sample up to $1000^{\circ} \mathrm{C}$ with minimal local temperature variation. We use atomic-resolution STEM ADF imaging operated at $80 \mathrm{keV}$ to track the stacking sequence change in bilayer $\mathrm{MoS}_{2}$. STEM ADF images show the 2D projection of atomic positions of the layered material, and the intensity in STEM ADF images is closely related to the atomic number and thickness of the material. Since Mo and $S$ have significantly different atomic numbers (Mo: 42 and S: 16), STEM ADF imaging is an ideal tool to identify the atomic species at different lattice positions, thus revealing the atomic structure of single- and few-layer $\mathrm{MoS}_{2}$. High-resolution STEM $\mathrm{ADF}$ imaging has been used to visualize the atomic arrangement in single- and few-layer $\mathrm{MoS}_{2}$, including stacking sequences, grain boundaries, point defects, edge sites, and even phase transformation. ${ }^{15,20,21,23,24}$ In our study, the electron beam serves two functions: (1) it facilitates in situ imaging of local atomic structure and changes thereof in bilayer $\mathrm{MoS}_{2} ;$ (2) it provides energy (in addition to the high temperature thermal bath) to stimulate local atomic movement. To make this atomic movement slow enough for real-time STEM tracking, an electron dose as low as $7 \times 10^{6}$ electrons/ $\mathrm{nm}^{2} \mathrm{~s}$ is applied. Although the electron energy of $80 \mathrm{keV}$ is close to the threshold for $S$ vacancy formation in monolayer $\mathrm{MoS}_{2}$, ${ }^{25}$ we are still able to image the regions of interest in bilayer samples for over $3 \mathrm{~min}$. Movies composed of continuous STEM ADF imaging allows the transition between different stacking sequences to be recorded.

In our in situ STEM study, we start from a CVD-grown pristine $\mathrm{MoS}_{2}$ bilayer with a uniform stacking sequence of $\mathrm{AB}$ or $\mathrm{AA}^{\prime}$. Transitions between different stacking sequences are observed at 350 and $400{ }^{\circ} \mathrm{C}$. Below, we focus on the representative case where the $\mathrm{AB}$ stacking is locally transformed into $\mathrm{AA}^{\prime}$ stacking at $400{ }^{\circ} \mathrm{C}$. Different stacking sequences are identified by comparing the experimental STEM ADF image with multislice simulation results, as shown in Figure 2d,e (see the Supporting Information for details). There are three distinct lattice points in the original $\mathrm{AB}$ stacked bilayer $\mathrm{MoS}_{2}$ (Figure 1): (1) the highest intensity corresponds to the 2D projection of the Mo-S-S column (with Mo from the top layer and S-S from the bottom layer); (2) the second highest intensity corresponds to the Mo column from the bottom layer; and (3) the weakest intensity corresponds to the S-S column projected from the top layer. When viewed from the top (Figure 1), the Mo-S-S columns and Mo columns are configured in a hexagonal lattice with 3-fold symmetry, while the hollow centers of the hexagonal lattice are occupied by the $S-S$ columns. As the electron beam scans in the AB stacked region (as shown in Figure $2 \mathrm{a}, \mathrm{d}$ ) for $14 \mathrm{~s}$, local atomic rearrangement occurs (marked by an orange ellipse in Figure 2b). Strikingly, after another $14 \mathrm{~s}$ of scanning in the same area, a triangular region with $\mathrm{AA}^{\prime}$ stacking appears as shown in Figure $2 c$,e. In this case, the three stacking boundaries between $\mathrm{AA}^{\prime}$ and $\mathrm{AB}$ stacked regions form extremely rapidly and stay stationary as the scan continues.

In contrast, stacking boundaries can also be induced more gradually by using a lower electron beam intensity. Here, to 

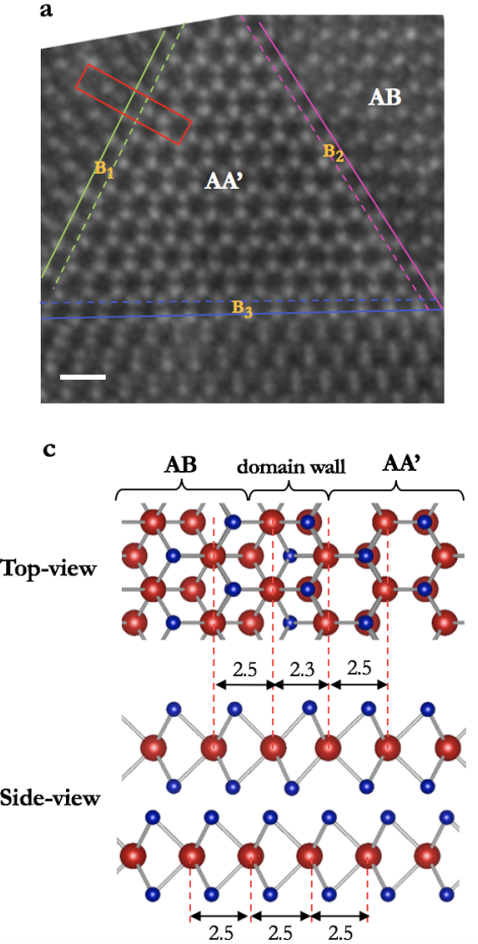

b

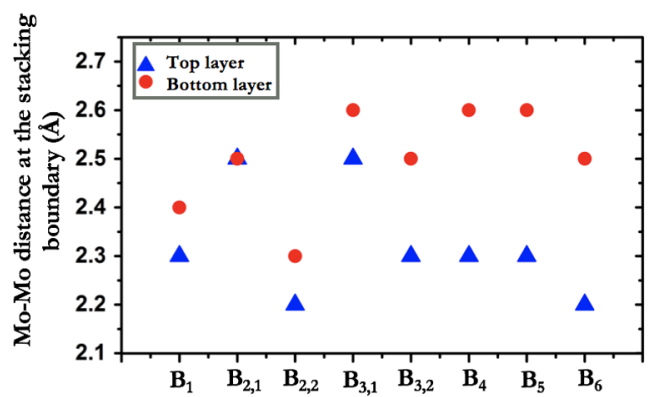

d

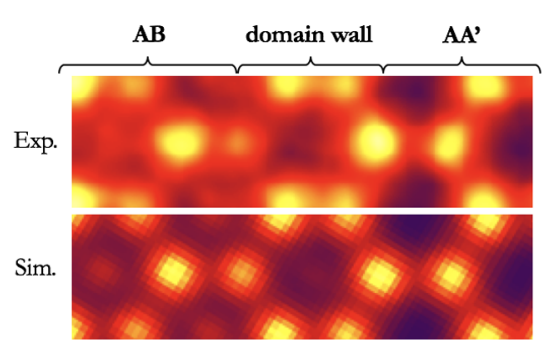

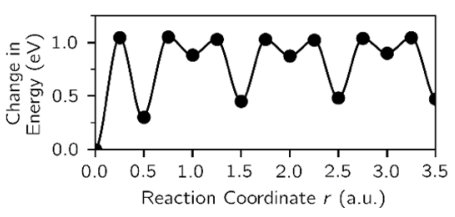

f

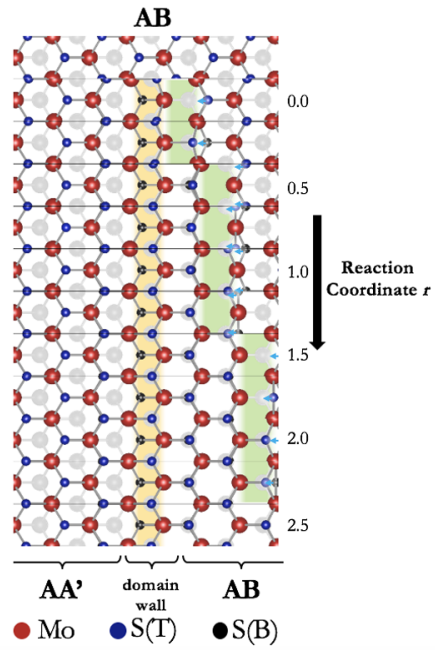

Figure 4. Atomic structure at the stacking boundaries. (a) A typical triangular $\mathrm{AA}^{\prime}$ stacked domain is connected to the $\mathrm{AB}$ stacked domain by atomically sharp boundaries. At the three boundaries (labeled as $B_{1}, B_{2}, B_{3}$ ), Mo lattices from the top layer are outlined by solid lines, and those from the bottom layer are outlined by dashed lines. Scale bar is $5 \AA$. (b) Summary of Mo lattice distances measured at six boundaries of two different samples in which $\mathrm{AA}^{\prime}$ and $\mathrm{AB}$ domains are stitched together (details of the boundary locations and labels can be found in the Supporting Information). Blue triangles represent the Mo lattice distances from the top layer, and red dots represent the Mo lattice distances from the bottom layer. The Mo lattice distances in the top layer at these boundaries shrink more than those in the bottom layer, indicating more severe structural change and thus more energy injection from the electron beam into the top layer. (c) Schematic of the boundary structure which is derived from DFT calculations and matches the experimentally observed most dominant boundary structure. This boundary structure features a T-phase-like structure. (d) Experimental STEM ADF image at a typical stacking boundary, with the intensity averaged over 5 unit cells across the boundary, compared to the simulated STEM ADF image based on the atomic model in (c). The experimental and simulated STEM ADF images are plotted on the same color scale. (e) Energy barrier per atom as $\mathrm{S}$ migrates during domain wall propagation. (f) Schematic diagram of domain wall nucleation ( $r$ $=0.0)$ and domain growth $(r>0.0)$, starting from a pristine, strained AB-stacked bilayer. See the Supporting Information Movie that depicts this process.

study the dynamics of the transition between stacking sequences, continuous STEM ADF images are recorded as shown in Figure 3. Figure 3a-d shows the evolution of a fixed area in the bilayer $\mathrm{MoS}_{2}$ sample in increments of 7 s. As a position reference between frames, red dotted circles mark the same atom from panel (a) to (d) in Figure 3, while dashed red triangles connect the same three lattice points from panel (a) to (h) in Figure 3. It is instructive to consider the nucleation of the stacking boundaries. Panels (a) and (e) in Figure 3 (Figure $3 \mathrm{e}$ is an enlarged image of the purple-squared area in Figure 3a) show the original $\mathrm{AB}$ stacked bilayer $\mathrm{MoS}_{2}$ at $t=0 \mathrm{~s}$. At $t=7 \mathrm{~s}$, local atomic rearrangement and a relative shift between the two $\mathrm{MoS}_{2}$ layers are observed (Figure 3b,f). The shift leads to the linear features as seen at the lower left corner of Figure $3 \mathrm{~b}$ (enclosed in a pink box). The separation between these linear features (orange lines in Figure $3 \mathrm{f}$ ) is $2.7 \pm 0.05 \AA$, which is equivalent to the single-layer Mo lattice periodicity $\boldsymbol{a}_{\mathbf{1}}\left(=\frac{\sqrt{3}}{2} \boldsymbol{a}\right.$, Figure 1). The simulated STEM ADF image (Supporting Information, Figure 3) shows that a relative in-plane displacement $^{1} \boldsymbol{u}=\frac{1}{3} \boldsymbol{a}_{1}$ agrees well with the experiment. The lateral shift between layers can be a source of large local strain, which may trigger other structural rearrangement or defects. For example, for a local region, we observe obvious shrinkage of the projected Mo-Mo distance (green lines in Figure 3f). This lattice distortion is most likely caused by $\mathrm{S}$ vacancy line defects which have also been observed in single-layer $\mathrm{MoS}_{2}$ samples under $\mathrm{TEM}^{27,28}$ due to the "knock-on" effect by the electron beam, as the electron beam's energy $(80 \mathrm{keV})$ is close to the threshold for $S$ vacancy formation in single-layer $\mathrm{MoS}_{2}$. Interstitial atoms and missing S-S columns are also observed in Figure 3f, as indicated by white arrows and blue arrows, respectively. These interstitial atoms and vacancies are highly mobile under combined electron irradiation and thermal heating, and eventually lead to the nucleation of $\mathrm{AA}^{\prime}$ stacked region shown in Figure $3 g$ (blue hexagons). A straight stacking boundary (the orange line in Figure $3 \mathrm{~g}$ ) is formed between $\mathrm{AA}^{\prime}$ and $\mathrm{AB}$ stacked regions. We also notice that, once the $\mathrm{AA}^{\prime}$ stacked domain nucleates, the interlayer shift (which originally concentrates at the lower left corner in Figure $3 b$ ) significantly reduces. Therefore, it is likely that the nucleation and formation of the $\mathrm{AA}^{\prime}$ stacked region release the strain caused by the interlayer shift, and the decrease in elastic energy compensates for the energy gain due to the newly formed stacking boundaries.

The $\mathrm{AA}^{\prime}$ stacked region grows under continuous electron beam irradiation, from $\sim 2.88 \mathrm{~nm}^{2}$ in Figure $3 \mathrm{c}$ to $\sim 4.75 \mathrm{~nm}^{2}$ in Figure $3 \mathrm{~d}$ after $7 \mathrm{~s}$. At this point, a second straight stacking 


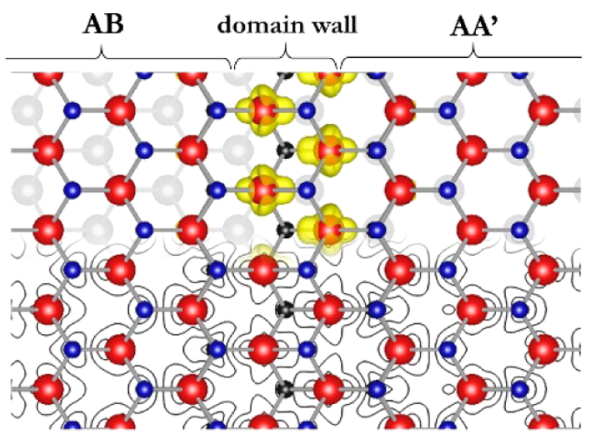

b

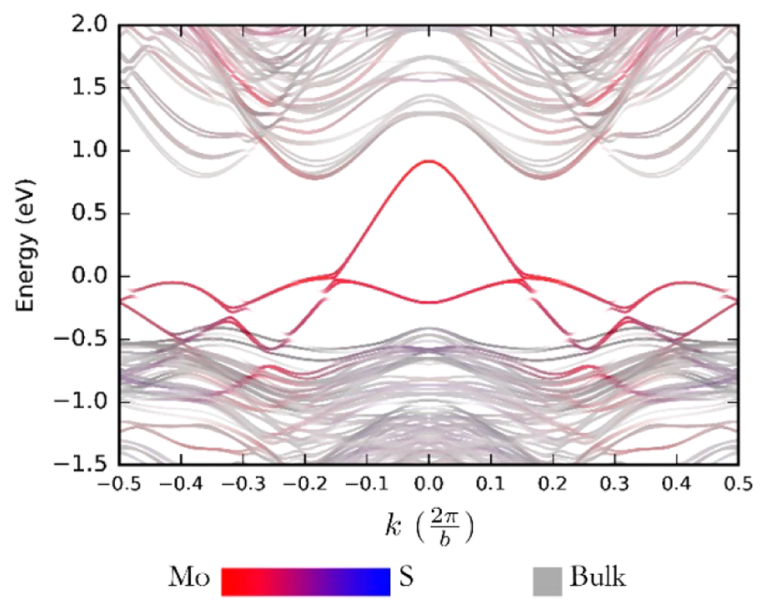

Figure 5. First-principles modeling of domain walls. (a) Atomic structure of the domain wall. The left side shows the AB domain, while the right side shows the $\mathrm{AA}^{\prime}$ domain, separated by the domain wall. The top half of the figure shows both the top and the bottom layers of the bilayer, superposed with the $15 \%$ isosurface of the charge densities of the boundary states. The bottom half of the figure shows only the top layer, superposed with the contour lines of the same charge densities on a two-dimensional cut of the Mo plane. (b) Band structure of the domain wall within DFT in the local density approximation (LDA).

boundary on the right (Figure $3 \mathrm{~h}$ ) forms at $60^{\circ}$ with respect to the left boundary. Since the corner where these two boundaries meet is pinned by some defects, the $\mathrm{AA}^{\prime}$ stacked region cannot expand upward and has to grow downward in the direction indicated by vector $W$. It is worth noting that, when we image and heat the as-grown $\mathrm{AA}^{\prime}$ stacked bilayer $\mathrm{MoS}_{2}$ under the same conditions, triangular $\mathrm{AB}$ stacked domains can also nucleate and grow from the originally $\mathrm{AA}^{\prime}$ stacked region (Supporting Information, Figure 4). This two-way transition between $\mathrm{AA}^{\prime}$ and $\mathrm{AB}$ stacked bilayer $\mathrm{MoS}_{2}$ suggests that the difference in total energy between $\mathrm{AA}^{\prime}$ and $\mathrm{AB}$ stacked bilayer $\mathrm{MoS}_{2}$ is very small, and excitations such as thermal energy and electron irradiation can overcome the energy barrier to allow the transition between these two stacking sequences. It is worth noting that we did not observe stacking boundary formation at a lower temperature such as $300{ }^{\circ} \mathrm{C}$ or a higher temperature ( $>400{ }^{\circ} \mathrm{C}$, where Mo and $\mathrm{S}$ atoms can be knocked out by the electron beam easily). This indicates the thermal annealing at a certain temperature provides the right amount of energy to assist the motion of $S$ atoms (which is the key step for the stacking sequence transition) while maintaining the crystal framework. In addition, we always observe stacking sequence transition seconds or tens of seconds after we start imaging an area, although we have let the sample stay at a certain temperature for a while $(>30 \mathrm{~min})$ before the imaging. This indicates the local interaction between the electron beam and the sample during the STEM imaging is the trigger for the stacking sequence transition.

We now examine the atomic structure of the stacking boundaries between the $\mathrm{AB}$ and $\mathrm{AA}^{\prime}$ stacked regions (Figure $4 a)$. The atomic structure shown in the STEM images of these boundaries has three main characteristics: (1) Mo lattices are continuous from the $\mathrm{AA}^{\prime}$ stacked region to the $\mathrm{AB}$ stacked region, which means that the Mo atoms in both regions remain roughly at their original positions; (2) the hollow centers of the hexagonal lattices at the boundary are occupied by fewer $S$ atoms compared to the original $\mathrm{AB}$ stacked bilayer $\mathrm{MoS}_{2}$, as shown in the experimental STEM ADF image in Figure 4d; (3) the Mo-Mo distance near the boundary has decreased. These observations are possible because Mo-S-S columns and Mo columns in $\mathrm{AB}$ stacked bilayer $\mathrm{MoS}_{2}$ show different intensities in $\mathrm{ADF}$ images. We then can differentiate the Mo atoms in the top layer from those in the bottom layer based on this intensity difference. In Figure 4a, three solid lines connect the Mo lattice points at the boundaries in the top layer (labeled as $B_{1}, B_{2}$, and $B_{3}$, respectively), while the dashed lines connect the Mo lattice points at the boundaries in the bottom layer. The Mo-Mo distances of both layers at these boundaries are shorter than that of bulk $\mathrm{MoS}_{2}$, which has also been observed in monolayer $\mathrm{MoS}_{2}$ with $\mathrm{S}$ line defects. ${ }^{27,28}$ Averaged over six boundaries (see the Supporting Information, Figure 5 for the boundary locations), the average Mo-Mo distance at the stacking boundaries is $\sim 2.3 \AA$ in the top layer, and $\sim 2.5 \AA$ in the bottom layer (Figure $4 \mathrm{~b}$ ). This indicates the top layer and bottom layer may experience different energetics due to electron-matter interaction.

To further quantify and understand the exact atomic structure at the stacking boundaries, we perform first-principles DFT calculations ${ }^{29}$ on different boundary structures (see the Supporting Information for computational details). The boundary structure in Figure $4 \mathrm{c}$ best satisfies the three experimentally observed characteristics mentioned above, and is stabilized by having a T-phase-like structure. The simulated STEM ADF image of this boundary structure agrees very well with the experimental results (Figure 4d). Moreover, our calculations show that compressive strain due to Mo-Mo distance shrinkage favors the formation of $S$ vacancies (see the Supporting Information). This indicates that the nucleation of this T-phase-like boundary probably originates from the formation of S line defects, which are commonly observed in single-layer $\mathrm{MoS}_{2}$ under extensive electron beam irradiation. ${ }^{27,28}$ To understand each step of this boundary formation, we rely on DFT calculations to identify the minimum-energy path for the nucleation and growth of the $\mathrm{AA}^{\prime}$ stacked region from the original $\mathrm{AB}$ stacking (Figure $4 \mathrm{f}$, only top $\mathrm{MoS}_{2}$ layer is shown in color). Here, we use $r$ to represent the reaction coordinate of domain growth, and the energy change associated with each step is plotted in Figure 4e. At $r=0.0$, a domain wall 
(shaded orange in Figure 4f) is formed by ejecting the top $S$ atoms (blue balls) in the top $\mathrm{MoS}_{2}$ layer. This leaves the bottom $S$ atoms (black balls) exposed. Subsequent domain growth is achieved by the migration of the nearest $S$ atoms in the top $\mathrm{MoS}_{2}$ layer toward the domain wall. This process only involves $S$ atoms in the top layer of bilayer $\mathrm{MoS}_{2}$, because the energy absorbed by the bottom $\mathrm{MoS}_{2}$ layer from the electron beam is significantly reduced due to the shielding effect by the top layer. ${ }^{30}$ The minimum-energy path for the $S$ migration in the top $\mathrm{MoS}_{2}$ layer is composed of two steps: (1) the top $\mathrm{S}$ atoms of the top layer migrating first $(r=0.25)$, followed by (2) the bottom $S$ atoms of the same layer migrating $(r=0.5)$, which is also a local energy minimum (Figure 4e). At this point, a unit cell of $\mathrm{AA}^{\prime}$ stacking is formed (Figure 4f). Subsequent expansion of the $\mathrm{AA}^{\prime}$ region involves the repetition of the above two $S$ migration steps (Figure $4 \mathrm{f}$ ). Since the energy barrier for each migration step is small, ranging from 0.15 to $1.05 \mathrm{eV}$ per atom (Figure 4e), this domain growth process can be easily activated by the $80 \mathrm{keV}$ electron beam used in the experiment. This $\mathrm{S}$ migrating process has also been previously reported for the $\mathrm{H}$-to- $\mathrm{H}^{\prime}$ transition in monolayer $\mathrm{MoS}_{2}{ }^{24}$ which is also observed in our experiment (Supporting Information, Figure 2a,b). Notably, this inversion domain formation in monolayer $\mathrm{MoS}_{2}$ alone cannot explain our experimentally obtained atomically sharp stacking boundaries, which require both domain inversion in the top layer via $S$ migrating and bonding modification in the bottom layer at the boundaries (Figure 4 and Supporting Information, Figure 2c). This indicates a strong interlayer interaction is the key to forming atomically sharp stacking boundaries in bilayer $\mathrm{MoS}_{2}$. We emphasize the novel integration of different inversion symmetries in the same bilayer $\mathrm{MoS}_{2}$ sample via creation of atomically sharp stacking boundaries between different stacking sequences.

Finally, we calculate the electronic band structure of the boundary and find in-gap states localized at the domain wall (see the Supporting Information for details). To identify states from the boundary, we project the band structure onto atomic wave functions of atoms near the stacking boundary (Figure $5 a)$. In the band structure (Figure $5 \mathrm{~b}$ ), states with contributions from Mo and $S$ atoms adjacent to the domain wall are colored red and blue, respectively, while contributions from atoms in the bulk are colored gray. We see that, importantly, the presence of the domain wall introduces metallic in-gap states that are absent in either $\mathrm{AA}^{\prime}$ or $\mathrm{AB}$ stacked $\mathrm{MoS}_{2}$ bilayer. Similar in-gap states have also been observed at the edges ${ }^{23,31}$ and mirror twin boundaries ${ }^{20,23,32}$ of monolayer $\mathrm{MoS}_{2}$. The ingap states in our case are composed primarily of $4 d$-states from Mo atoms bordering the domain wall. From the contour lines of the integrated charge density in Figure 5a, we see that these in-gap states are exponentially localized at the domain wall. We note that DFT-LDA has a well-known tendency to underestimate band gaps and overestimate occupied bandwidths. ${ }^{33}$ Appropriate theory of higher levels such as the GW approximation $^{34-36}$ must be used to obtain quantitatively accurate quasiparticle band structures. Here, LDA already provides a good description of qualitative features and wave function character.

In summary, we have demonstrated a novel way to engineer domains with different inversion symmetries in bilayer $\mathrm{MoS}_{2}$ through the synergy of thermal excitation and electron irradiation. Using our method, we are able to reversibly convert between regions of $\mathrm{AA}^{\prime}$ and $\mathrm{AB}$ stacking, thus switching between stacking sequences that result in different valley polarization. Through a first-principles study, we find that there are highly localized metallic states at the atomically sharp stacking boundaries. We propose that this approach may be used to reversibly pattern regions of different inversion symmetries, as well as controllably embed defect lines with different transport properties, within $2 \mathrm{D}$ semiconductors. This method of domain and symmetry engineering can be generalized to other TMDCs. We hope that this study will inspire greater interest in future studies of valleytronics control and defect state patterning in atomically thin $\mathrm{MoS}_{2}$ and other TMDCs via nanoscale symmetry engineering.

\section{ASSOCIATED CONTENT}

\section{S Supporting Information}

The Supporting Information is available free of charge on the ACS Publications website at DOI: 10.1021/acs.jpcc.7b08398.

Experimental methods for CVD growth of $\mathrm{MoS}_{2}$, TEM sample preparation, and STEM ADF imaging and multislice simulation. Identification of $\mathrm{AB}$ and $\mathrm{AA}^{\prime}$ stacked bilayer $\mathrm{MoS}_{2}$ regions by comparing the experimentally collected and simulated STEM ADF images. Experimentally observed phase transition from $\mathrm{H}$ to $\mathrm{H}^{\prime}$ in monolayer $\mathrm{MoS}_{2}$ and interlayer interaction in bilayer $\mathrm{MoS}_{2}$. The formation of linear features in $\mathrm{AB}$ stacked bilayer $\mathrm{MoS}_{2}$ due to the relative shift between the layers. Experimental observation of transition from $\mathrm{AA}^{\prime}$ stacking to $\mathrm{AB}$ stacking in bilayer $\mathrm{MoS}_{2}$. Measurement of Mo-Mo distance at the stacking boundaries between $\mathrm{AA}^{\prime}$ and $\mathrm{AB}$ stacked domains. Details for first-principles calculations, including calculations of different stacking boundary structures between $\mathrm{AA}^{\prime}$ and $\mathrm{AB}$ stacked bilayer $\mathrm{MoS}_{2}$ domains, band structures at these boundaries with and without $\mathrm{H}$ passivation, and the formation energy for $S$ vacancy (PDF)

Movie depicting domain wall nucleation and domain growth (MPG)

Movie of continuous STEM ADF imaging showing the transition between different stacking sequences (MPG)

\section{AUTHOR INFORMATION}

\section{Corresponding Author}

*E-mail: azettl@berkeley.edu. ORCID 을

Aiming Yan: 0000-0002-5394-7498

Diana Y. Qiu: 0000-0003-3067-6987

\section{Author Contributions}

A.Z. and A.Y. conceived and designed the experiment. A.Y. collected and analyzed scanning transmission electron microscopy (STEM) data. C.S.O., D.Y.Q., and S.G.L. performed density functional theory calculations. A.Y. and C.M. synthesized $\mathrm{MoS}_{2}$ samples. C.O. wrote the Matlab code for multislice simulations. C.O. and J.C. contributed to and provided advice for STEM data collection and analysis. A.Y., A.Z., C.S.O., D.Y.Q., and S.G.L. co-wrote the manuscript. All authors discussed the results and commented on the paper.

\section{Notes}

The authors declare no competing financial interest.

\section{ACKNOWLEDGMENTS}

This work was supported in part by the Director, Office of Science, Office of Basic Energy Sciences, Materials Sciences and 
Engineering Division, of the U.S. Department of Energy under Contract No. DE-AC02-05-CH11231, within the sp2-bonded Materials Program (KC2207) which provided for STEM data collection and DFT calculations, and within the van der Waals Heterostructures Program (KCWF16) which provided for analysis of the STEM data; by the Molecular Foundry of the Lawrence Berkeley National Laboratory, supported by the Office of Science, Office of Basic Energy Sciences, of the U.S. Department of Energy under Contract No. DE-AC0205CH11231 which provided for additional STEM instrumentation; by the National Science Foundation, under Grant No. 1542741, which provided for sample growth and preparation, under Grant No. DMR-1206512, which provided for development of the sample transfer method, and under Grant No. DMR-1508412, which provided for supplementary DFT calculations. Computational resources have been provided by the NSF through XSEDE resources at SDSC. C.S.O. acknowledges support from the Singapore National Research Foundation (Clean Energy) PhD Scholarship. We acknowledge Chengyu Song in the Molecular Foundry of the Lawrence Berkeley National Laboratory for TEM technical support.

\section{REFERENCES}

(1) Alden, J. S.; Tsen, A. W.; Huang, P. Y.; Hovden, R.; Brown, L.; Park, J.; Muller, D. A.; McEuen, P. L. Strain Solitons and Topological Defects in Bilayer Graphene. Proc. Natl. Acad. Sci. U. S. A. 2013, 110, $11256-11260$

(2) Butz, B.; Dolle, C.; Niekiel, F.; Weber, K.; Waldmann, D.; Weber, H. B.; Meyer, B.; Spiecker, E. Dislocations in Bilayer Graphene. Nature 2014, 505, 533-537.

(3) Ju, L.; Shi, Z.; Nair, N.; Lv, Y.; Jin, C.; Velasco, J., Jr; OjedaAristizabal, C.; Bechtel, H. A.; Martin, M. C.; Zettl, A. Topological Valley Transport at Bilayer Graphene Domain Walls. Nature 2015, 520, 650-655.

(4) Zhang, F.; MacDonald, A. H.; Mele, E. J. Valley Chern Numbers and Boundary Modes in Gapped Bilayer Graphene. Proc. Natl. Acad. Sci. U. S. A. 2013, 110, 10546-10551.

(5) Vaezi, A.; Liang, Y.; Ngai, D. H.; Yang, L.; Kim, E.-A. Topological Edge States at a Tilt Boundary in Gated Multilayer Graphene. Phys. Rev. X 2013, 3, 21018.

(6) Xiao, D.; Liu, G.-B.; Feng, W.; Xu, X.; Yao, W. Coupled Spin and Valley Physics in Monolayers of MoS 2 and Other Group-VI Dichalcogenides. Phys. Rev. Lett. 2012, 108, 196802.

(7) Mak, K. F.; He, K.; Shan, J.; Heinz, T. F. Control of Valley Polarization in Monolayer MoS2 by Optical Helicity. Nat. Nanotechnol. 2012, 7, 494-498.

(8) Zeng, H.; Dai, J.; Yao, W.; Xiao, D.; Cui, X. Valley Polarization in MoS2Monolayers by Optical Pumping. Nat. Nanotechnol. 2012, 7, 490-493.

(9) Mak, K. F.; McGill, K. L.; Park, J.; McEuen, P. L. The Valley Hall Effect in MoS2 Transistors. Science (Washington, DC, U. S.) 2014, 344, 1489-1492.

(10) Lee, J.; Mak, K. F.; Shan, J. Electrical Control of the Valley Hall Effect in Bilayer MoS2 Transistors. Nat. Nanotechnol. 2016, 11, 421425 .

(11) Jiang, T.; Liu, H.; Huang, D.; Zhang, S.; Li, Y.; Gong, X.; Shen, Y.-R.; Liu, W.-T.; Wu, S. Valley and Band Structure Engineering of Folded MoS2 Bilayers. Nat. Nanotechnol. 2014, 9, 825-829.

(12) van der Zande, A. M.; Kunstmann, J.; Chernikov, A.; Chenet, D. A.; You, Y.; Zhang, X.; Huang, P. Y.; Berkelbach, T. C.; Wang, L.; Zhang, F. Tailoring the Electronic Structure in Bilayer Molybdenum Disulfide via Interlayer Twist. Nano Lett. 2014, 14, 3869-3875.

(13) Huang, S.; Ling, X.; Liang, L.; Kong, J.; Terrones, H.; Meunier, V.; Dresselhaus, M. S. Probing the Interlayer Coupling of Twisted Bilayer MoS2 Using Photoluminescence Spectroscopy. Nano Lett. 2014, 14, 5500-5508.
(14) Suzuki, R.; Sakano, M.; Zhang, Y. J.; Akashi, R.; Morikawa, D.; Harasawa, A.; Yaji, K.; Kuroda, K.; Miyamoto, K.; Okuda, T. ValleyDependent Spin Polarization in Bulk MoS2 with Broken Inversion Symmetry. Nat. Nanotechnol. 2014, 9, 611-617.

(15) Yan, A.; Chen, W.; Ophus, C.; Ciston, J.; Lin, Y.; Persson, K.; Zettl, A. Identifying Different Stacking Sequences in Few-Layer CVDGrown MoS2 by Low-Energy Atomic-Resolution Scanning Transmission Electron Microscopy. Phys. Rev. B: Condens. Matter Mater. Phys. 2016, 93, 041420.

(16) Lu, X.; Utama, M.; Lin, J.; Luo, X.; Zhao, Y.; Zhang, J.; Pantelides, S. T.; Zhou, W.; Quek, S. Y.; Xiong, Q. Rapid and Nondestructive Identification of Polytypism and Stacking Sequences in Few-Layer Molybdenum Diselenide by Raman Spectroscopy. Adv. Mater. 2015, 27, 4502-4508.

(17) Liu, K.; Zhang, L.; Cao, T.; Jin, C.; Qiu, D.; Zhou, Q.; Zettl, A.; Yang, P.; Louie, S. G.; Wang, F. Evolution of Interlayer Coupling in Twisted Molybdenum Disulfide Bilayers. Nat. Commun. 2014, 5, 4966.

(18) Xia, M.; Li, B.; Yin, K.; Capellini, G.; Niu, G.; Gong, Y.; Zhou, W.; Ajayan, P. M.; Xie, Y.-H. Spectroscopic Signatures of AA' and AB Stacking of Chemical Vapor Deposited Bilayer MoS2. ACS Nano 2015, 9, 12246-12254.

(19) He, J.; Hummer, K.; Franchini, C. Stacking Effects on the Electronic and Optical Properties of Bilayer Transition Metal Dichalcogenides MoS2, MoSe2, WS2, and WSe2. Phys. Rev. B: Condens. Matter Mater. Phys. 2014, 89, 075409.

(20) van der Zande, A. M.; Huang, P. Y.; Chenet, D. A.; Berkelbach, T. C.; You, Y.; Lee, G.-H.; Heinz, T. F.; Reichman, D. R.; Muller, D. A.; Hone, J. C. Grains and Grain Boundaries in Highly Crystalline Monolayer Molybdenum Disulphide. Nat. Mater. 2013, 12, 554-561.

(21) Najmaei, S.; Liu, Z.; Zhou, W.; Zou, X.; Shi, G.; Lei, S.; Yakobson, B. I.; Idrobo, J.-C.; Ajayan, P. M.; Lou, J. Vapour Phase Growth and Grain Boundary Structure of Molybdenum Disulphide Atomic Layers. Nat. Mater. 2013, 12, 754-759.

(22) Suk, J. W.; Kitt, A.; Magnuson, C. W.; Hao, Y.; Ahmed, S.; An, J.; Swan, A. K.; Goldberg, B. B.; Ruoff, R. S. Transfer of CVD-Grown Monolayer Graphene onto Arbitrary Substrates. ACS Nano 2011, 5, 6916-6924.

(23) Zhou, W.; Zou, X.; Najmaei, S.; Liu, Z.; Shi, Y.; Kong, J.; Lou, J.; Ajayan, P. M.; Yakobson, B. I.; Idrobo, J.-C. Intrinsic Structural Defects in Monolayer Molybdenum Disulfide. Nano Lett. 2013, 13, 26152622.

(24) Lin, Y.-C.; Dumcenco, D. O.; Huang, Y.-S.; Suenaga, K. Atomic Mechanism of the Semiconducting-to-Metallic Phase Transition in Single-Layered MoS2. Nat. Nanotechnol. 2014, 9, 391-396.

(25) Komsa, H.-P.; Kotakoski, J.; Kurasch, S.; Lehtinen, O.; Kaiser, U.; Krasheninnikov, A. V. Two-Dimensional Transition Metal Dichalcogenides under Electron Irradiation: Defect Production and Doping. Phys. Rev. Lett. 2012, 109, 035503.

(26) Kirkland, E. J. Advanced Computing in Electron Microscopy; Springer Science \& Business Media: New York, 2010.

(27) Wang, S.; Lee, G.-D.; Lee, S.; Yoon, E.; Warner, J. H. Detailed Atomic Reconstruction of Extended Line Defects in Monolayer MoS2. ACS Nano 2016, 10, 5419-5430.

(28) Komsa, H.-P.; Kurasch, S.; Lehtinen, O.; Kaiser, U.; Krasheninnikov, A. V. From Point to Extended Defects in TwoDimensional MoS2: Evolution of Atomic Structure under Electron Irradiation. Phys. Rev. B: Condens. Matter Mater. Phys. 2013, 88, 035301.

(29) Giannozzi, P.; Baroni, S.; Bonini, N.; Calandra, M.; Car, R.; Cavazzoni, C.; Ceresoli, D.; Chiarotti, G. L.; Cococcioni, M.; Dabo, I. QUANTUM ESPRESSO: A Modular and Open-Source Software Project for Quantum Simulations of Materials. J. Phys.: Condens. Matter 2009, 21, 395502.

(30) Zan, R.; Ramasse, Q. M.; Jalil, R.; Georgiou, T.; Bangert, U.; Novoselov, K. S. Control of Radiation Damage in MoS2 by Graphene Encapsulation. ACS Nano 2013, 7, 10167-10174.

(31) Pan, H.; Zhang, Y.-W. Edge-Dependent Structural, Electronic and Magnetic Properties of MoS2 Nanoribbons. J. Mater. Chem. 2012, 22, 7280 . 
(32) Zou, X.; Liu, Y.; Yakobson, B. I. Predicting Dislocations and Grain Boundaries in Two-Dimensional Metal-Disulfides from the First Principles. Nano Lett. 2013, 13, 253-258.

(33) Northrup, J. E.; Hybertsen, M. S.; Louie, S. G. Theory of Quasiparticle Energies in Alkali Metals. Phys. Rev. Lett. 1987, 59, 819822.

(34) Hybertsen, M.; Louie, S. Electron Correlation in Semiconductors and Insulators: Band Gaps and Quasiparticle Energies. Phys. Rev. B: Condens. Matter Mater. Phys. 1986, 34, 5390-5413.

(35) Qiu, D. Y.; da Jornada, F. H.; Louie, S. G. Optical Spectrum of MoS 2: Many-Body Effects and Diversity of Exciton States. Phys. Rev. Lett. 2013, 111, 119901.

(36) Qiu, D. Y.; da Jornada, F. H.; Louie, S. G. Screening and ManyBody Effects in Two-Dimensional Crystals: Monolayer MoS 2. Phys. Rev. B: Condens. Matter Mater. Phys. 2016, 93, 235435. 